\title{
25 Research Square \\ The Potentials of Calotropis procera against Filarial Elephantiasis: An in- silico approach
}

\section{Aswin Mohan}

University of Kerala

Shanitha Shaji

University of Kerala

Sunitha $\mathbf{P}$

University of Kerala

Shahanas Naisam

Accubits Invent Pvt. Ltd.

Nidhin Sreekumar ( $\nabla$ nidhin@accubits.com )

Accubits Technologies Inc. https://orcid.org/0000-0001-9345-4471

\section{Research Article}

Keywords: Lymphatic filariasis, Brugia malayi, Calotropis procera, Molecular Docking, Molecular Dynamics

Posted Date: April 22nd, 2021

DOl: https://doi.org/10.21203/rs.3.rs-418805/v1

License: (c) (i) This work is licensed under a Creative Commons Attribution 4.0 International License. Read Full License 


\section{Abstract}

Lymphatic filariasis is one of the major diseases that belong to the category of neglected tropical illness. Filarial nematodes are the cause of the disease and are transmitted to humans via blood-feeding arthropod vectors. Drugs such as Albendazole, Ivermectin and diethylcarbamazine are administered either individually or in combination to overcome the progress of the lymphatic filariasis. However, these drugs have some disadvantages like temporary hair loss, dizziness, nausea etc. The filarial parasites have multifunctional proteins including the Glutathione-s-transferase (GST) enzyme which plays a major role in detoxification of endogenous electrophilic compounds. This study aims at the identification of a natural molecule that has the potential to bind with the GST enzyme and thus interrupt the detoxification process within the filarial parasite, Brugia malayi. A medicinal plant Calotropis procera, owing to its anthelmintic properties was searched for the presence of potential phytocompounds. The phytocompounds were docked against the homology modeled GST enzyme using the MOE software. The results were screened and analyzed based on the Lipinski rule of 5 . N-octanoate was the phytocompound obtained based on molecular docking, subjected to molecular dynamics. These results require further in vitro and in vivo validation to consider n-octanoate as a potential drug candidate for lymphatic filariasis treatment.

\section{Introduction}

Lymphatic filariasis or Filariasis is one of the neglected tropical diseases caused by the nematode filarial parasites Wuchereria bancrofti, Brugia malayi and Brugia timori. Filariasis causes permanent limb disability and can even cause permanent malformations of several body parts (Ottesen et al. 2008). The filarial parasites are thread-like parasitic nematodes (roundworms) and are transmitted to the human host through blood-feeding arthropod vectors. The adult worms reside in specific tissues within the host where they release thread-like microfilariae into the blood. The circulating microfilariae enter the vector during blood-feeding and develop into an infective larva ready for human transmission. The intensity of the disease in the host depends on the locations of the tissue preferred by the microfilariae and matured worms. The matured worms inhabit lymph vessels, causing lymphatic inflammation and dysfunction due to blockage and host reactions and gradually lead to lymphedema and fibrosis (Stillwaggon et al. 2016). Prolonged and recurrent infection with these worms can lead to a buildup of excess tissue in the affected area, as in elephantiasis (Cross 1996). The present drugs have poor inhibition on adult parasitic worms. Current treatment includes administration of drugs individually or in combination, latter showing better results (Brophy and Pritchard 1994).

Published articles on filariasis emphasize the importance of Glutathione-s-transferases (GST) in chronic infections. GSTs are multifunctional proteins that belong to an enzyme family which filarial parasitic worms depend for their survival in the host (Bhargavi et al. 2005). GSTs are major detoxification enzymes that neutralize cytotoxic compounds and protect cells and tissues from the reactive oxygen species attack. They catalyze the conjugation of Glutathione (GSH) to endogenous electrophilic compounds that are less harmful (Bhoj et al. 2020). These enzymes protect the tissue from damage caused by free 
radicals, superoxides and aids in the intracellular transport of non-catalytic carrier proteins (Sommer et al. 2001). GST's involvement in drug resistance and biosynthesis of arachidonic acid metabolites makes GST a more attractive target for therapeutics (Van Ommen et al. 1991. Studies prove that GST of humans are structurally different from worms and can be used as a potential target for developing antifilarial drugs (Brophy et al. 2000).

Calotropis procera Linn. is a common shrub mostly found as a weed around India (lyadurai et al. 2020), attributed to warm and dry areas, the sub- Himalayan regions and the southern regions of the country. Different parts of Calotropis procera have been reported to possess different therapeutic activities such as proteolytic (Atal and Sethi 1962) antimicrobial, larvicidal (Al-Rowaily et al. 2020), nematocidal, anticancer, anti-inflammatory (Basu and Chaudhuri 1991; Kumar and Basu 1994). Its flowers possess digestive and tonic properties.

The mechanism of action and side effects of the drugs used in Filariasis treatment are presented in Table 1.

\section{Materials And Methods}

This study focuses on proposing a potent lead molecule against filarial elephantiasis from traditional medicinal plant Calotropis procera. Homology modeling, Molecular docking and molecular dynamics studies are performed on a potential drug target of filarial parasite - Glutathione-s-transferase with phytocompounds retrieved from Calotropis procera.

\section{Target Selection}

The sequence of Glutathione s transferase of Brugia malayi was retrieved from UniProt (UniProt ID: A0AOH5S7P0) (2021) having 208 residues. The sequence is uploaded in the swiss model tool for finding templates for homology modeling. Homology modeling is a modeling technique by which atomic resolution models of protein are designed and it can be either from homologous proteins or ab initio (from the scratch). The protein was modeled using an online tool - swiss model (Peitsch, 1996). The sequence was uploaded in the swiss model and ran a search for homologous protein templates. Template $5 \mathrm{~d} 73.1$.A is selected from the results having $100 \%$ sequence identity and the values GMQE: 0.95, QMEAN: -0.11, QSQE: 1.00 showed that template 5 d73.1.A was a suitable model. The structure of Glutathione $S$ transferase of Brugia malayi had modeled using template 5d73.1.A and the structure was an artificial construct like parasitic nematodes with a single chain - A chain with a total length of 208 residues starting from MET and ends with GLN. In this study, the binding sites selected for docking were retrieved from literatures and the residues are Tyr7, Phe8, Gly12, Leu13, Trp 38, Lys42, GIn49, Leu50, Ser 63, Arg95, His98, Thr99, Tyr 101, Thr102, Tyr106 and Val202 (Domadia, et al., 2008, Kalani, et al., 2014 and Mathew, et al., 2011).

\section{Target Validation}


The homology modeled structure was validated using multiple tools like ERRAT, Verify3D and Procheck. (Colovos and Yeates. 1993; Laskowski et al. 1993). The modeled protein was uploaded in the SAVESv6.0 (ERRAT - DOE-MBI Structure Lab UCLA) to estimate the ERRAT, Verify 3D and Procheck plots. ERRAT identifies and calculates the statistics of non-bonded interactions between different atom types and generates "the error function versus position of a 9-residue sliding window" plot based on the data from highly refined structures. Verify 3D determines the compatibility of an atomic model (3D) by using its own amino acid sequence (1D). A structural class based on the atomic model's location and environment (alpha, beta, loop, polar, nonpolar, etc.) is assigned and compared with the results of the best structures. Procheck analyzes residue-by-residue geometry and overall structure geometry (Ramachandran plot, chi1chi2 plot, main-chain parameters, side chain parameters, residue properties, main-chain bond lengths, main chain bond angles and planar groups) of modelled structure to provide the stereochemical quality of the protein.

\section{Ligand Preparation}

Phytocompound composition of Calotropis procera were collected from literature survey and the respective structures were obtained from PubChem (Kim et al. 2021). The structures were optimized and preprocessed using MOE (Molecular Operating Environment) tool.

\section{ADME Prediction}

ADME (Absorption, Digestion, Metabolism and Elimination) properties of the phytocompounds were calculated using Swiss ADME. Swiss ADME calculated the ALogp, MLogP, WLogP, Molecular weight, Solubility, GI (Gastrointestinal) absorption, and blood-brain barrier permeability of the selected phytocompounds. The canonical smiles of the phytocompounds retrieved from PubChem were given as an input for ADME prediction. Physicochemical properties of ligands were studied using DataWarrior.

\section{Molecular Docking}

Molecular docking studies were performed in MOE (Inc.2016). Pre-docking procedures such as protein preparation, binding site identification and ligand preparation were done prior to docking. All the heteroatoms were extracted during protein preparation and polar hydrogens were added. The binding sites retrieved from literature were defined before docking. The prepared phytocompound and the protein were subjected to docking with OPLS- AA force field. Post docking evaluation of the outcome of each docking pose were analyzed individually. The 2D representation of the ligand-bound complex was used to check the Hydrogen- bonds, based on favorable H-bond interactions and S-score of the docked complex, phytocompounds were filtered. Hydrogen bond interactions between residues of defined binding site and phytocompounds were considered as favorable H-bond interactions. Phytocompounds that did not possess favorable $\mathrm{H}$-bond interactions were filtered off. Molecular properties (Molecular weight, AlogP, $\mathrm{H}-$ bond donors and $\mathrm{H}$-bond acceptors) and toxicity of these ligands were estimated and those ligands that did not satisfy the rule of 5 of Lipinski were filtered off. The ligands with mutagenic properties were filtered, the binding affinity of ligands were estimated and analyzed for better interactions. 


\section{Molecular Dynamics}

For molecular dynamics, GROMACS (version 2020.1), an open-source molecular dynamics software, was used (Berendsen et al. 1995). There was a separation of the docked complex into protein and ligand. The force field used was the CHARMM36 all-atom force field, and the CGenFF server supported the ligand parameters and topologies compatible with the above force field. The separated protein and ligand were developed into a complex and ligand topology and parameter files were added to the protein topology file, resulting in the topology file of the complex. In the solvation process the complex was solvated using water. In order to prepare the complex for energy minimization, required ions were added into the solvated complex. Energy minimization was carried out in order to bring the complex into its minimum potential energy. Restraining the ligand and treatment of temperature coupling groups was accompanied by energy minimization. The complex was regulated by equilibration of volume- NVT ensemble (constant Number of particles, Volume, and Temperature) followed by equilibration of pressure- NPT ensemble (Number of particles, Pressure, and Temperature). An MD simulation run for 40 ns was initiated. The trajectories of docked complexes were analyzed through 40ns of molecular dynamics simulation. The simulated complex was analyzed for Root Mean Square Deviation, Root Mean Square Fluctuation, Radius of gyration, number of hydrogen bonds and interaction energy.

\section{Result And Discussion}

Glutathione-s-transferase (GST) is the multifunctional protein that comprises major detoxification enzymes. It mainly helps the organism's macromolecules to stay intact from the attack of electrophiles and they catalyze the conjugation of Glutathione to the electrophiles. They are mostly found in the cytosol and in addition to catalyzing the conjugation to electrophiles they perform some peroxidase and isomerase activities. Since it has a significant role in the parasite worm's life cycle, it can be considered as a drug target.

Sequence from UniProt was used to search for homologous protein templates, and template 5d73.1.A was selected as it had $100 \%$ sequence identity and the following parameter values; GMQE (Global Model Quality Estimation): 0.95, QMEAN: -0.11, QSQE (Quaternary Structure Quality Estimate): 1.00 showed that template 5d73.1.A was a suitable model.

ERRAT plots show a quality factor of 93.9547 which shows that the model has a good quality (Messaoudi et al. 2013). 96.14\% of the residues had passed the average 3D-1D score $>=0.2$ when validated by Verify3D (Ullah et al. 2012). In the Ramachandran plot, $0 \%$ residues were found in the disallowed region inferring that the model has a good secondary structure (Waghmare et al. 2016)

Glutathione-s-transferase was taken as the target and phytocompounds structures from the plant Calotropis procera were taken as ligands for docking studies. For docking purposes, the Molecular Operating Environment was used as the platform to study the interactions. The protein preparation was done for eliminating the water molecules and adding explicit hydrogens. Binding sites were defined and as follows Tyr7, Phe8, Gly12, Leu13, Trp 38, Lys 42, Gln49, Leu50, Ser63, Arg95, His98, Thr99, Tyr101, 
Thr102, Tyr106 and Val202. Ligands were prepared in MOE and the ligands and protein were subjected to docking.

The docking result had 4057 poses and out of which 49 poses were filtered based on favorable H-bond interactions and S score (Table 2). Molecular properties of these 44 ligands were listed with the help of ADMET descriptors and toxicity prediction tools (SwissADME and MOE). From the list, 16 ligands satisfying Lipinski's rule of 5 and with non-mutagenic (non-toxic) properties were taken to the next level (Table 3) i.e., for binding affinity prediction. Binding affinity is the strength of binding interaction of a biomolecule to ligand/ binding partner.

Phytocompound n-Octanoate showed desired hydrogen bond interaction with the given binding sites such as Trp 38, Lys 42, Gln49 and exhibit druggable characteristics (Table 4). The 2D plot of interactions and hydrophobic interactions of n-Octanoate with Glutathione-s-transferase was studied (Fig. 1). This ligand has the least binding affinity; hence it has the highest binding stability with the target protein (Glutathione-s-transferase). The ligand n-Octanoate resulted in better docking scores and satisfied Lipinski's rule of 5 .

The docked complex was subjected to molecular dynamics using GROMACS. The temperature was equilibrated at $300 \mathrm{~K}$ and the pressure was equilibrated at $1 \mathrm{bar}$ for a time for MD run, $40 \mathrm{~ns}$. The RMSD (Fig. 2(a)) plot and RMSF (Root mean square fluctuation) plot (Fig. 2(b)) of the complex after simulation were obtained. The RMSD deviates in between $0.1 \mathrm{~nm}$ to $0.2 \mathrm{~nm}$, stabilizes at $0.15 \mathrm{~nm}$ from $20 \mathrm{~ns}$ up to the end of simulation, showing less deviation in RMSD (Cob-Calan et al. 2019). The part of the protein that fluctuates mostly during the period of simulation can be understood from RMSF, infers in the flexibility of protein in a binding state. Residue 208 shows the greater fluctuation among the total protein residues. Radius of Gyration defines the rigidity of the system, greater degree of fluctuation results in the inconsistency throughout the simulation shows the compactness of the system. The RoG of the complex fluctuated in between $1.7 \mathrm{~nm}$ and $1.75 \mathrm{~m}$, with an average of $1.725 \mathrm{~nm}$ and found more fluctuating at 25 ns inferring in the higher compactness/ rigidity of the system (Fig. 2(c)) (Turner et al. 2019). The total number of hydrogen bonds in the complex throughout the simulation was also estimated and a maximum of 182 hydrogen bonds, a minimum of 140 hydrogen bonds, an average of 165 bonds were found to be in the complex (Fig. 2(d)). Interaction energies of the complex including Lennard- Jones short-range and Coulombic short-range potential energies were calculated. The Lennard-Jones shortrange potential energy of the complex was found to be $-31.5848 \mathrm{~kJ} / \mathrm{mol}$ with a total drift of 53.4165 $\mathrm{KJ} / \mathrm{mol}$ (Fig.3(a)) and Coulombic shot-range potential energy, $-6.63557 \mathrm{~kJ} / \mathrm{mol}$ with a total drift of $9.61891 \mathrm{KJ} / \mathrm{mol}$ (Fig. 3(b)).

In the future, several factors will be playing a prominent role in the prevalence of lymphatic filariasis. A study suggests that changing patterns of climate will be interfering in the spread of parasitic diseases. A re-emergence of parasitic diseases is possible due to global warming and its associated changes in the environment (Rodó et al. 2013; Wu et al. 2016). The migration to metropolitan cities and international trade / Global market can be a potential channel to spread transmittable diseases (Irvine et al. 2015). 
Mass Drug Administration (MDA) is controlling the disease to an extent (Kalyanasundaram et al. 2020). Mutation of parasitic nematodes can decrease the efficiency of MDA (Kwarteng et al. 2016). Additional measures like morbidity management, vector control and surveillance must be employed more efficiently to minimize the spread of the disease (Famakinde 2018) and (Irvine et al. 2015) Vector control, reducing human - vector contact and improvising the standard of living can be crucial in eliminating filariasis (Rebollo and Bockarie 2017).

\section{Conclusion}

The docking studies using Glutathione-S- transferase of Brugia malayi as the target intend to find a potent drug candidate for Lymphatic Filariasis. phytocompounds from the plant Calotropis procera were used as the ligand molecules. The phytocompounds 9-Decanoate and n-Octanoate have shown promising results and can be considered for the purpose. These ligands have $\mathrm{H}$-bond interaction with the binding sites of GST. 9-decanoate and n-octanoate showed the least binding energies $-4.1 \mathrm{kcal} / \mathrm{mol}$ and $-4.9 \mathrm{kcal} / \mathrm{mol}$ respectively from the screened phytocompound database. Further in vitro and in vivo studies can be used to confirm these candidates as a potential drug that can be used against Filariasis.

\section{Declarations}

Funding: Not applicable.

Conflicts of interest/Competing interests: The authors declare that they have no conflicting financial interests.

Availability of data and material: Not applicable.

Code availability: Not applicable.

Authors' contributions (optional: please review the submission guidelines from the journal whether statements are mandatory)

Additional declarations for articles in life science journals that report the results of studies involving humans and/or animals: Not applicable.

Ethics approval: Not applicable.

Consent to participate: Not applicable.

Consent for publication: Not applicable.

\section{Acknowledgements}

I am grateful to Dr. Achuthsankar S. Nair, Head of the Department, Department of Computational Biology and Bioinformatics, University of Kerala for rendering all the facilities for the completion of the work. I am 
particularly thankful for Mr. Vinod M. P., Ms. Chinchu E. R., Mr. Ashish Sudharshan, Ms. Roshny Prasad, Dept. of Computational Biology \& Bioinformatics, University of Kerala for the support and encouragement.

\section{References}

Al-Rowaily SL., Abd-ElGawad, A.M., Assaeed, A.M., Elgamal, A.M., Gendy, A.E.-N.G.E., Mohamed, T.A., Dar, B.A., Mohamed, T.K. and Elshamy, A.I. (2020). Essential Oil of Calotropis procera: Comparative Chemical Profiles, Antimicrobial Activity, and Allelopathic Potential on Weeds. Molecules, 25(21), p.5203.

Atal C, Sethi P (1962) Proteolytic Activity of Some Indian Plants. Planta Med 10:77-90.

Basu A, Chaudhuri AKN (1991) Preliminary studies on the antiinflammatory and analgesic activities of Calotropis procera root extract. J Ethnopharmacol 31:319-324

Berendsen, H. J. C., van der Spoel, D., \& van Drunen, R. (1995). GROMACS: A message-passing parallel molecular dynamics implementation. Computer Physics Communications, 91(1-3), 43-56.

Bhargavi R, Vishwakarma S, Murty US (2005) Modeling analysis of GST (glutathione-S-transferases) from Wuchereria bancrofti and Brugia malayi. Bioinformation 1:25

Bhoj, P.S., Bahekar, S., Khatri, V., Singh, N., Togre, N.S., Goswami, K., Chandak, H.S. and Dash, D. (2020). Role of Glutathione in Chalcone Derivative Induced Apoptosis of Brugia malayi and its Possible Therapeutic Implication. Acta Parasitologica.

Brophy PM, Pritchard DI (1994) Parasitic helminth glutathione S-transferases: an update on their potential as targets for immuno-and chemotherapy. Exp Parasitol 79:89-96

Brophy PM, Campbell AM, van Eldik AJ, et al (2000) $\beta$-Carbonyl substituted glutathione conjugates as inhibitors of O. volvulus GST2. Bioorg Med Chem Lett 10:979-981

Cob-Calan, N.N., Chi-Uluac, L.A., Ortiz-Chi, F., Cerqueda-García, D., Navarrete-Vázquez, G., Ruiz-Sánchez, E. and Hernández-Núñez, E. (2019). Molecular Docking and Dynamics Simulation of Protein $\beta$-Tubulin and Antifungal Cyclic Lipopeptides. Molecules, 24(18), p.3387.

Cross JH (2015) Filarial Nematodes *. In: Manual of Clinical Microbiology, 10th Edition. American Society of Microbiology, pp 2212-2221

Domadia PN, Bhunia A, Sivaraman J, et al (2008) Berberine targets assembly of Escherichia coli cell division protein FtsZ. Biochemistry 47:3225-3234

Famakinde DO (2018) Mosquitoes and the lymphatic filarial parasites: research trends and budding roadmaps to future disease eradication. Trop Med Infect Dis 3:4 
Irvine MA, Reimer LJ, Njenga SM, et al (2015) Modelling strategies to break transmission of lymphatic filariasis-aggregation, adherence and vector competence greatly alter elimination. Parasit Vectors 8:1-19

lyadurai, R., Gunasekaran, K., Jose, A. and Pitchaimuthu, K. (2020). Calotropis poisoning with severe cardiac toxicity A case report. Journal of Family Medicine and Primary Care, 9(8), p.4444.

Kalani K, Kushwaha V, Sharma P, et al (2014) In vitro, in silico and in vivo studies of ursolic acid as an anti-filarial agent. PLoS One 9:e111244

Kalyanasundaram, R., Khatri, V. and Chauhan, N. (2020). Advances in Vaccine Development for Human Lymphatic Filariasis. Trends in Parasitology, 36(2), pp.195-205.

Kim, S., Chen, J., Cheng, T., Gindulyte, A., He, J., He, S., Li, Q., Shoemaker, B.A., Thiessen, P.A., Yu, B., Zaslavsky, L., Zhang, J. and Bolton, E.E. (2021). PubChem in 2021: new data content and improved web interfaces. Nucleic Acids Research, [online] 49(D1), pp.D1388-D1395. Available at: https://academic.oup.com/nar/article/49/D1/D1388/5957164.

Kumar VL, Basu N (1994) Anti-inflammatory activity of the latex of Calotropis procera. J Ethnopharmacol $44: 123-125$

Kwarteng A, Ahuno ST, Akoto FO (2016) Killing filarial nematode parasites: role of treatment options and host immune response. Infect Dis poverty 5:1-6

Laskowski RA, MacArthur MW, Moss DS, Thornton JM (1993) PROCHECK: a program to check the stereochemical quality of protein structures. J Appl Crystallogr 26:283-291

Mathew N, Srinivasan L, Karunan T, et al (2011) Studies on filarial GST as a target for antifilarial drug development-in silico and in vitro inhibition of filarial GST by substituted 1, 4-naphthoquinones. J Mol Model 17:2651-2657

Messaoudi A, Belguith H, Hamida J Ben (2013) Homology modeling and virtual screening approaches to identify potent inhibitors of VEB-1 $\beta$-lactamase. Theor Biol Med Model 10:1-10

Molecular Operating Environment (MOE), 2019.01; Chemical Computing Group ULC, 1010 Sherbooke St. West, Suite \#910, Montreal, QC, Canada, H3A 2R7, 2021

Ottesen EA, Hooper PJ, Bradley M, Biswas G (2008) The Global Programme to Eliminate Lymphatic Filariasis: Health Impact after 8 Years. PLoS Negl Trop Dis 2:e317.

Peitsch, M. C. (1996). ProMod and Swiss-Model: Internet-based tools for automated comparative protein modelling. Biochemical Society Transactions, 24(1), 274-279.

Rebollo MP, Bockarie MJ (2017) Can lymphatic filariasis be eliminated by 2020? Trends Parasitol 33:8392 
Rodó X, Pascual M, Doblas-Reyes FJ, et al (2013) Climate change and infectious diseases: can we meet the needs for better prediction? Clim Change 118:625-640

Sawers, L. and Stillwaggon, E. (2020). Economic Costs and Benefits of Community-Based LymphedemaManagement Programs for Lymphatic Filariasis in India. The American Journal of Tropical Medicine and Hygiene, 103(1), pp.295-302.

Sommer A, Nimtz M, Conradt HS, et al (2001) Structural Analysis and Antibody Response to the Extracellular Glutathione S-Transferases from Onchocerca volvulus. Infect Immun 69:7718-7728

Stillwaggon E, Sawers L, Rout J, et al (2016) Economic costs and benefits of a community-based lymphedema management program for lymphatic filariasis in Odisha State, India. Am J Trop Med Hyg 95:877-884

Turner, M., Mutter, S.T., Kennedy-Britten, O.D. and Platts, J.A. (2019). Molecular dynamics simulation of aluminium binding to amyloid- $\beta$ and its effect on peptide structure. PLOS ONE, 14(6), p.e0217992.

Ullah, M., Ghosh, T., Ishaque, N., Absar, N. and Hira, J. (2012). A Bioinformatics Approach for Homology Modeling Binding Site Identification of Triosephosphate Isomerase from Plasmodium falciparum 3D7. Journal of Young Pharmacists, 4(4), pp.261-266.

(2021) UniProt: the universal protein knowledgebase in 2021. Nucleic Acids Res 49:D480-D489. https://doi.org/10.1093/nar/gkaa1100.

Van Ommen B, Ploemen J, Bogaards JJP, et al (1991) Irreversible inhibition of rat glutathione Stransferase $1-1$ by quinones and their glutathione conjugates. Structure-activity relationship and mechanism. Biochem J 276:661-666

Waghmare, S., Buxi, A., Nandurkar, Y., Shelke, A. and Chavan, R. (2015). In silico sequence analysis, homology modeling and function annotation of leishmanolysin from Leishmania donovani. Journal of Parasitic Diseases, 40(4), pp.1266-1269.

Wu X, Lu Y, Zhou S, et al (2016) Impact of climate change on human infectious diseases: Empirical evidence and human adaptation. Environ Int 86:14-23

\section{Tables}

Due to technical limitations, the tables are only available as a download in the supplemental files section.

\section{Figures}




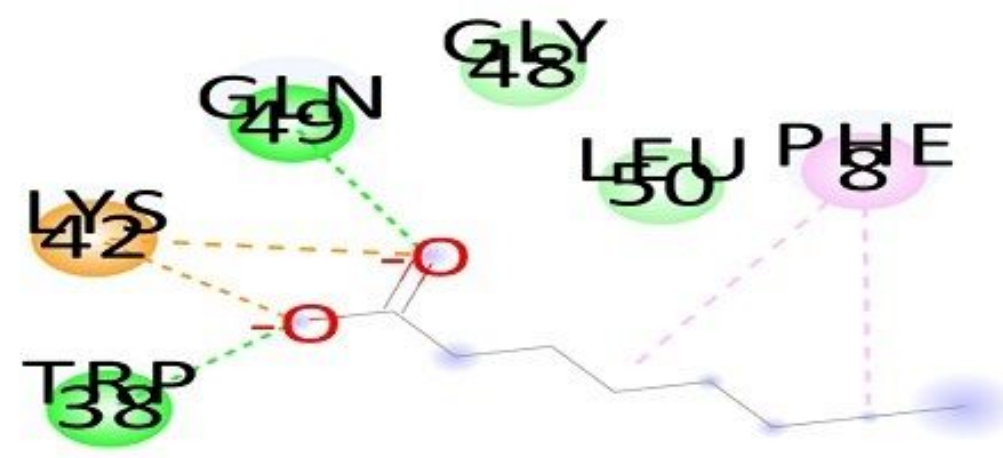

\section{Eby}

\section{T¥R HE}

\section{IXB}

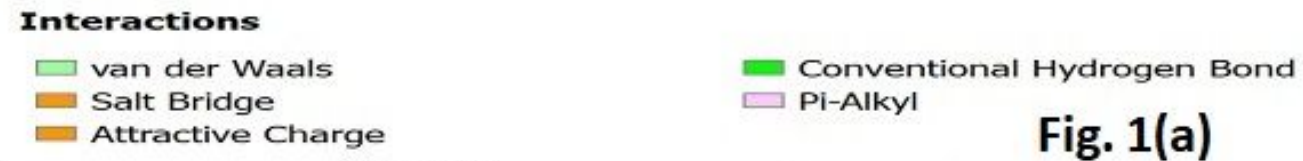

Fig. 1(a)

Figure 1

(a) 2D interactions of n-Octanoate with Glutathione-s-transferase, (b) Hydrophobic surface of interaction n-Octanoate with Glutathione-s-transferase 
RMSD

Backbone after lsg fit to Backbone

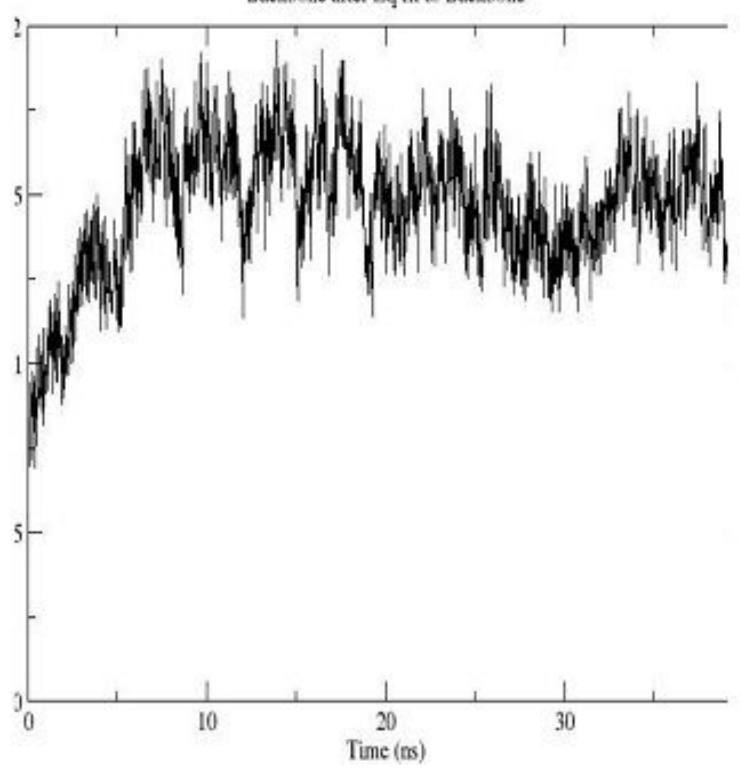

Fig. 2(a)

Radius of gyration (total and around axes)

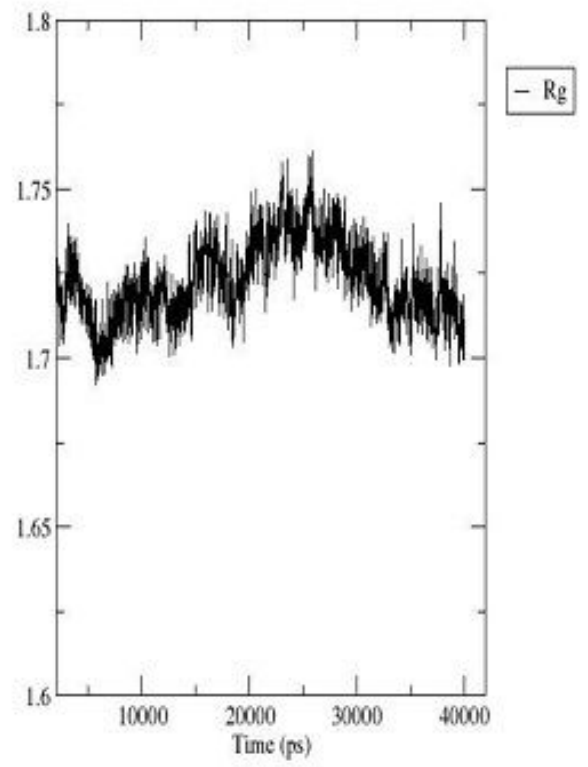

Fig. 2(c)
RMS fluctuation

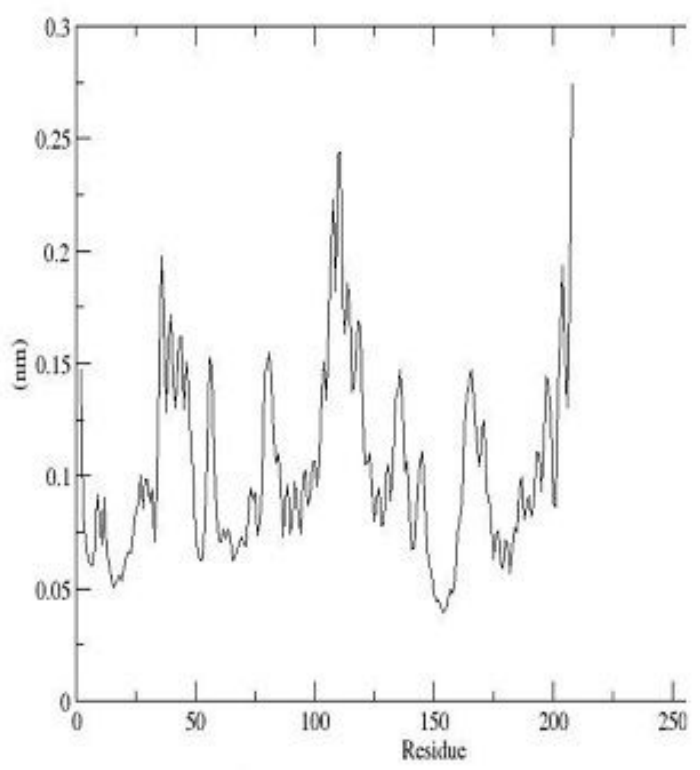

Fig. 2(b)

Hydrogen Bonds

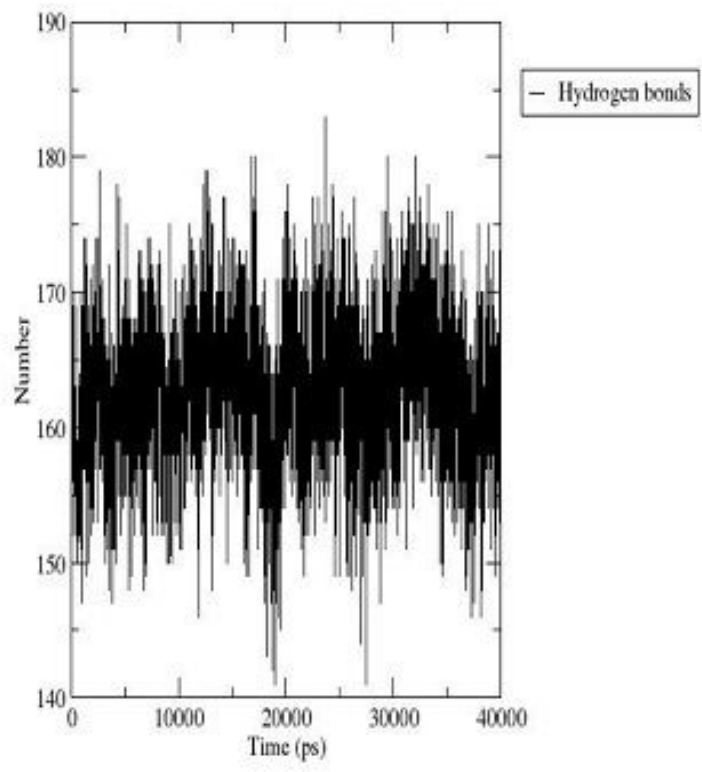

Fig. 2(d)

\section{Figure 2}

(a) RMSD plot of n-Octanoate - Glutathione-s-transferase complex, (b) RMSf plot of n-Octanoate Glutathione-s-transferase complex, (c) RoG plot of n-Octanoate - Glutathione-s-transferase complex, (d) Number of hydrogen bonds plot of n-Octanoate - Glutathione-s-transferase complex 


\section{GROMACS Energies}

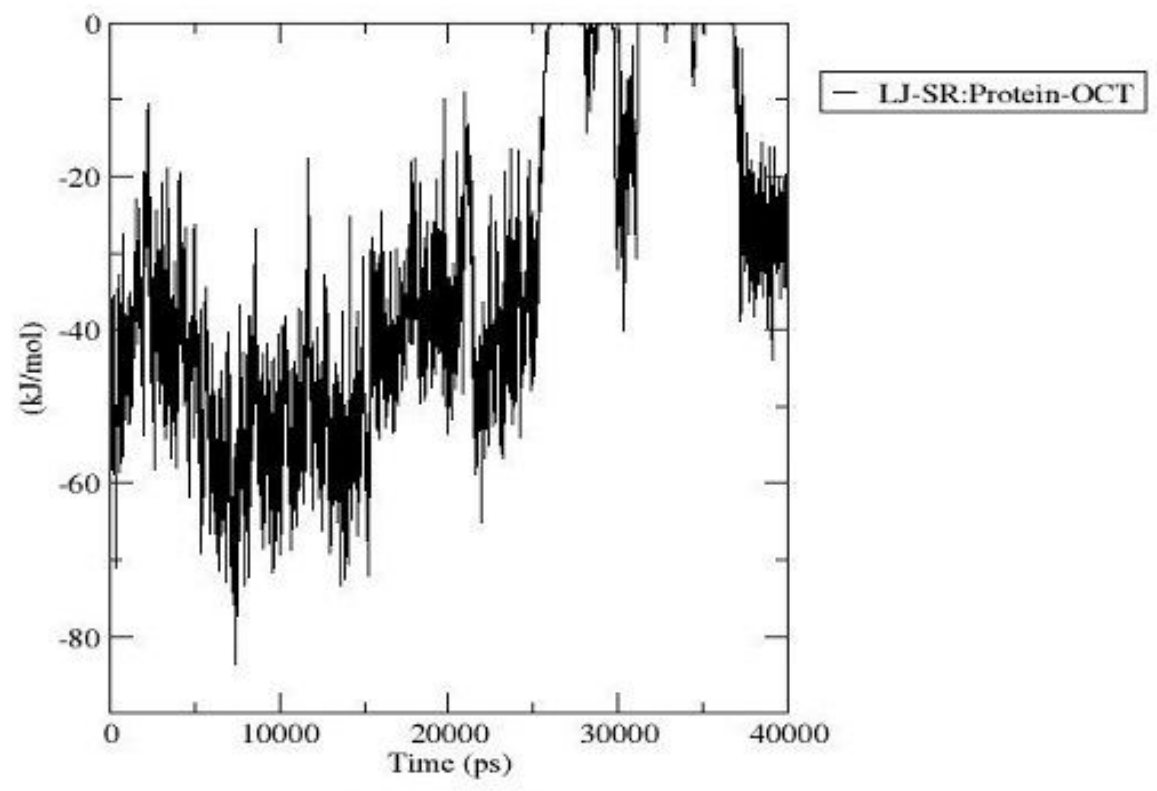

Fig. 3(a)

GROMACS Energies

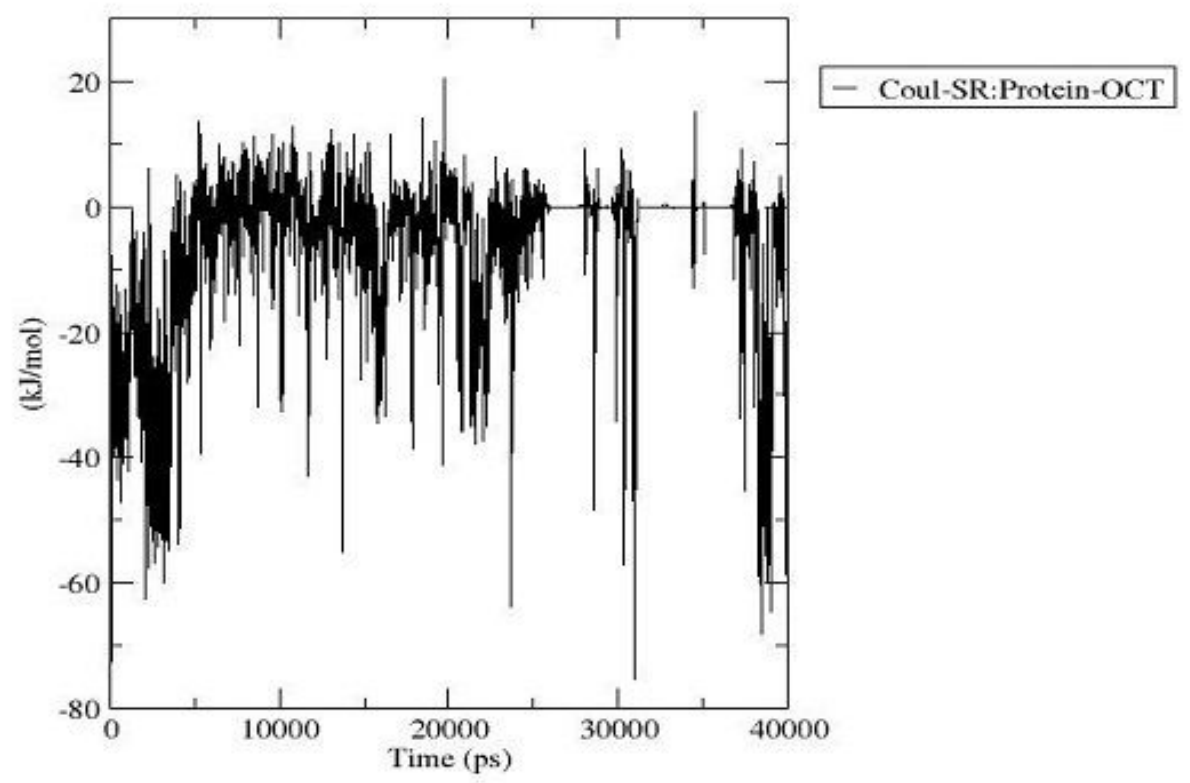

Fig. 3(b)

Figure 3

(a) Lennard-Jones short-range potential energy of n-Octanoate - Glutathione-s-transferase complex, (b) Coulombic Short-range potential energy of n-Octanoate - Glutathione-s-transferase complex

\section{Supplementary Files}


This is a list of supplementary files associated with this preprint. Click to download.

- Tables.pdf 